\title{
Probing the $4 \mathrm{f}$ states of ceria by tunneling spectroscopy
}

\author{
Xiang Shao, Jan-Frederik Jerratsch, Niklas Nilius* and Hans-Joachim Freund \\ Received 8th April 2011, Accepted 17th May 2011 \\ DOI: $10.1039 / \mathrm{c} 1 \mathrm{cp21113g}$
}

Low-temperature scanning tunneling microscopy and spectroscopy have been employed to analyze the local electronic structure of the (111) surface of a ceria thin film grown on $\mathrm{Ru}(0001)$. On pristine, defect-free oxide terraces, the empty $4 \mathrm{f}$ states of $\mathrm{Ce}^{4+}$ ions appear as the only spectral feature inside the $6 \mathrm{eV}$ oxide band gap. In contrast, occupied states are detected between -1.0 and $-1.5 \mathrm{eV}$ below $E_{\mathrm{Fermi}}$ in conductance spectra of different point and line defects, such as surface oxygen vacancies, grain boundaries and step edges. They are assigned to partially filled $4 \mathrm{f}$ states localized at the $\mathrm{Ce}^{3+}$ ions. The presence of excess electrons indicates the oxygen-deficient nature of the direct oxide environment. The f state spectroscopy with the STM allows us to probe the spatial distribution of $\mathrm{Ce}^{3+}$ ions in the ceria surface, providing unique insight into the local reduction state of this chemically important material system.

\section{Introduction}

The surface properties of oxides are largely governed by defects in their top-most layers. It is their availability and spatial arrangement that determines the nucleation behavior of ad-metals and hence the size and shape distribution of particles on the oxide surface. Defects are also preferred binding sites for gas-phase molecules and therefore inevitably connected with the chemical performance of the oxide material. Their electronic peculiarities are finally responsible for various optical and conductance phenomena, such as the appearance of discrete absorption and emission lines in UV/Vis spectroscopy and the low electrical resistance of different semi-conducting oxides.

The present knowledge on oxide defects largely derives from a rigorous application of electronic and vibrational spectroscopies, the latter using suited probe molecules to explore defect properties. ${ }^{1}$ Those techniques provide insight into the abundance of defects and disclose the associated changes in the oxide electronic structure. However, they are usually unable to identify the spatial distribution and atomic position of defects in the surface. This information can only be accessed if a certain defect position imprints a characteristic electronic, ${ }^{2}$ optical, ${ }^{3}$ or magnetic signature ${ }^{4}$ onto the averaging spectrum. The common way to map the spatial arrangement of defects in an oxide surface is the application of scanning probe techniques. ${ }^{5-8}$ This approach provides not only the atomic-scale position of individual defects, but may also elucidate their electronic properties by applying associated spectroscopic methods. ${ }^{6,9}$

In this work, scanning tunneling microscopy (STM) and spectroscopy have been employed to investigate the defect

Fritz-Haber-Institut der Max-Planck-Gesellschaft, Faradayweg 4-6, D-14195 Berlin,Germany.E-mail:nilius@fhi-berlin.mpg.de structure of crystalline $\mathrm{CeO}_{2}$ (111) films. Ceria has recently moved into the focus of research because of its outstanding performance in various oxidation reactions. ${ }^{10,11}$ The high catalytic activity is connected with the facile reducibility of this oxide and the concomitant ability to supply lattice oxygen for a reaction. In an equally facile manner, the $\mathrm{O}$ vacancies are healed again in an oxygen-rich ambience, making ceria the ideal buffer system for oxygen in a catalytic process. ${ }^{12}$

Oxygen vacancies in surface and subsurface ceria layers have been extensively studied with STM and scanning force microscopy, revealing the defect position in the oxide lattice and the associated relaxations of neighboring ions. ${ }^{13-16}$ The vacancies are mainly generated with two approaches, namely thermal treatment of the oxide to stimulate oxygen desorption ${ }^{13,14}$ and electron bombardment. ${ }^{17}$ In both cases, they were exclusively observed on plain oxide terraces, although DFT calculations found the defect-formation energy to be substantially lowered at low-coordinated step and corner sites. ${ }^{18-20}$ The presence of $\mathrm{O}$ vacancies along the ceria steps is also suggested from the preferred nucleation of ad-metals at those sites, as revealed from growth studies performed with $\mathrm{Au}$ and Ag. ${ }^{17,21}$ The fact that no defects could be identified directly along the edges so far might be related to problems in resolving such tiny lattice irregularities next to a corrugated step, and cannot be considered as a proof for the absence of defects at low-coordinated sites.

To overcome potential imaging problems, we have chosen an alternative approach to probe the defect distribution in the ceria surface. The creation of an $\mathrm{O}$ vacancy leaves behind two electrons that localize in split-off states of the initially empty $4 \mathrm{f}$ orbitals of adjacent $\mathrm{Ce}$ ions. Those states are located inside the ceria band gap at $-1.5 \mathrm{eV}$ below the Fermi level $\left(E_{\mathrm{F}}\right)$ and can easily be detected with photoelectron (PES) and electronenergy-loss spectroscopy (EELS). ${ }^{22,23}$ In this work, we use STM conductance spectroscopy to probe the filling of the Ce $4 \mathrm{f}$ 
levels upon $\mathrm{O}$ vacancy formation on the atomic scale. By this means, the local reduction state of the oxide surface can be monitored even if the associated defects leave no topographic traces in the STM images. While only single vacancies have been considered in an earlier paper, ${ }^{24}$ the full variety of point and line defects exposed on the ceria surface is described in this comprehensive study.

\section{Experiment}

The experiments were carried out in a custom-built ultra-high vacuum STM operated at liquid helium temperature. Surface imaging was performed in the constant current mode with electron currents below $5 \mathrm{pA}$ in order to enable tunneling through the insulating film. Electronic properties of the sample were deduced from differential conductance spectra measured with a lock-in technique and a disabled feed-back loop $(1073 \mathrm{~Hz}$, $10 \mathrm{mV} \mathrm{rms}$ ). Due to the non-conductive nature of bulk ceria, we have prepared thin oxide films on a $\mathrm{Ru}(0001)$ single crystal following a recipe by Mullins et al. ${ }^{17,25}$ The Ce metal was deposited in two steps from a Mo crucible onto the sputtered/ annealed Ru surface in $5 \times 10^{-7} \mathrm{mbar}$ of $\mathrm{O}_{2}$. Whereas the first step was performed at $100 \mathrm{~K}$ to prepare an oxide wetting layer, the second deposition was carried out at $450 \mathrm{~K}$ to promote ordering of the film. After another annealing cycle at $1000 \mathrm{~K}$ in $\mathrm{O}_{2}$, an atomically flat and crystalline $\mathrm{CeO}_{2}$ film was obtained. It is characterized by a sharp hexagonal $(\sqrt{ } 2 \times \sqrt{ } 2)$ LEED pattern, indicative of the low-energy $\mathrm{CeO}_{2}(111)$ surface (Fig. 1a). ${ }^{20}$ Corresponding STM images display extended ceria terraces of up to $50 \mathrm{~nm}$ diameter, being occasionally disrupted by holes and cracks that reach down to the metal support. We believe that these openings remain in the film after coalescence of adjacent oxide patches at high temperature. The formation of a (111)-oriented $\mathrm{CeO}_{2}$ surface is also compatible with atomically resolved STM images, as shown in Fig. 1b. At positive sample bias, a hexagonal array of protrusions with $3.9 \AA$-periodicity is observed that arises from tunneling into empty states of $\mathrm{Ce}$ ions in the top-most oxide trilayer. At negative bias, tunneling into the occupied $\mathrm{O} 2 \mathrm{p}$ levels governs the image contrast; however atomic resolution is more difficult to obtain. This is in contrast to earlier experiments performed at room or elevated temperature, where atomic

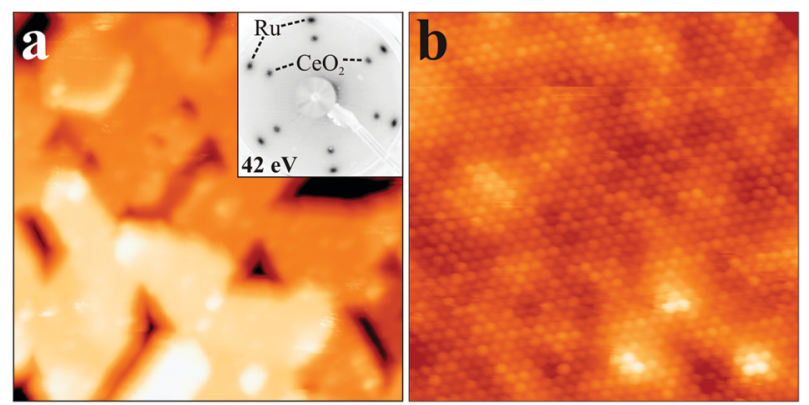

Fig. 1 (a) STM topographic image of a five-trilayer thick $\mathrm{CeO}_{2}$ film grown on $\operatorname{Ru}(0001)\left(3.0 \mathrm{~V}, 70 \times 70 \mathrm{~nm}^{2}\right)$. The inset shows the corresponding LEED pattern. (b) Atomically-resolved image showing the Ce sublattice $\left(1.2 \mathrm{~V}, 12 \times 12 \mathrm{~nm}^{2}\right)$. The paired protrusions in the lower part of the image are assigned to oxygen vacancies that give rise to the bright appearance of adjacent $\mathrm{Ce}^{4+}$ ions. resolution was mainly attained in filled-state images. ${ }^{14,16} \mathrm{We}$ suspect that tunneling out of the $\mathrm{O} 2 \mathrm{p}$ orbitals is hindered by the limited mobility of ceria electrons at the cryogenic temperature used in our experiment. The film thickness was varied between one and six $\mathrm{O}-\mathrm{Ce}-\mathrm{O}$ trilayers, corresponding to $3-18 \AA$.

\section{Results and discussion}

\subsection{Regular oxide surface}

The electronic structure of stoichiometric $\mathrm{CeO}_{2}$ was measured as a function of film thickness by means of STM conductance spectroscopy (Fig. 2). Whereas bare $\mathrm{Ru}(0001)$ shows the expected exponential increase of the $\mathrm{d} I / \mathrm{d} V$ signal with bias, a region of zero-conductance that marks the oxide band gap is detected on the oxide film. For thick films (above five tri-layers) the gap stretches from -2.5 to $+3.5 \mathrm{~V}$ and is hardly smaller than in bulk ceria $(6.3 \mathrm{eV})$. The gap size slightly shrinks with decreasing film thickness; however also the band onsets become less pronounced which renders a quantitative analysis difficult. A closer look at the $\mathrm{d} I / \mathrm{d} V$ curves reveals that the gap region is not featureless, but exhibits an additional peak at 2.3 to $2.5 \mathrm{~V}$ (Fig. 2). We assign this maximum to the empty Ce $4 \mathrm{f}$ band in correspondence with EELS and inverse PES data that fixed the band center at $4.4 \mathrm{eV}$ above the ceria valence band or at $2.0 \mathrm{eV}$ above $E_{\mathrm{F}}{ }^{22,23}$ The position of the empty $\mathrm{Ce} 4 \mathrm{f}$ band has later been confirmed with DFT calculations, ${ }^{26,27}$ although the exact band energy was found to depend on details of the computational approach. The fact that the spatially confined $f$ states can be probed with STM spectroscopy is surprising at the first glance, but might be rationalized by a certain intermixing between the $4 \mathrm{f}$ and the $\mathrm{O} 2 \mathrm{p}$ states in accordance with the covalent nature of ceria. The respective mixed orbitals are delocalized at the oxide surface and hence accessible for the tunneling electrons. Furthermore, no alternative states are available inside the gap region, forcing the tip to approach the surface until sufficient overlap with the empty Ce $4 \mathrm{f}$ band is achieved. We note that $5 \mathrm{f}$ states of metallic uranium have been detected by tunneling spectroscopy before. ${ }^{28}$

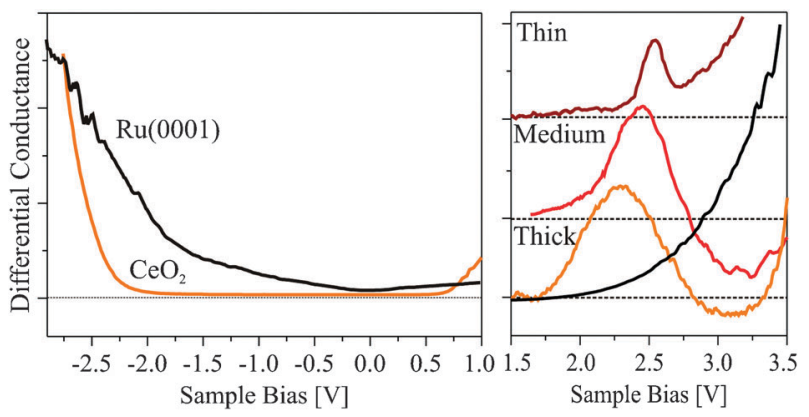

Fig. 2 Conductance spectra showing the filled (left, set point $-2.5 \mathrm{~V}$ ) and empty states (right, set point $+3.5 \mathrm{~V}$ ) of differently thick $\mathrm{CeO}_{2}$ films and the Ru support. Whereas the filled-state spectra are governed by the valence band onset of ceria at around $-2.5 \mathrm{~V}$, the empty-state spectra exhibit an extra peak at 2.25 to $2.75 \mathrm{~V}$ that is assigned to the empty $\mathrm{Ce} 4 \mathrm{f}$ band. The band broadens and down-shifts with increasing film thickness. Note the region of negative differential conductance at the high-side of the $\mathrm{f}$ derived $\mathrm{d} I / \mathrm{d} V$ peak. 
Two peculiarities of the $4 \mathrm{f}$ related spectral feature should be emphasized. First, the maximum at around $2.5 \mathrm{~V}$ is always accompanied by a region of negative $\mathrm{d} I / \mathrm{d} V$ signal at higher bias. This phenomenon is referred to as negative differential resistance (NDR) and often observed in the tunneling transport through energetically and spatially confined electronic states, e.g. molecular ${ }^{29}$ and atomic orbitals. ${ }^{30}$ It arises if a discrete transport channel closes at a certain bias and the associated conductivity loss is not compensated by the enhanced transmissibility of the tunnel barrier. In the present example, the NDR effect reflects the extra gap in the ceria states between the $\mathrm{f}$ levels and the conduction band onset, giving rise to a region of negative conductance. The second observation concerns the width of the f related $\mathrm{d} I / \mathrm{d} V$ maximum, which changes as a function of film thickness (Fig. 2). Whereas in a six-trilayer film, the full-width-at-half-maximum (FWHM) of the peak amounts to $600 \mathrm{mV}$, it decreases to 400 and $200 \mathrm{mV}$ in a triple and a single trilayer, respectively. In addition, the peak shifts away from the Fermi level when the film becomes thinner. We propose two reasons for the observed broadening, the first being inherent to the system and the second one relating to the measuring process. In ultrathin films, all Ce ions sit in identical positions and have the same $f$ state energy, while in thicker films $\mathrm{Ce}$ occupies interface, bulk-like and surface regions. These different environments give rise to small shifts of the $f$ band position, leading to an effective broadening of the band as seen in the spectra. Secondly, band positions in conductance spectroscopy are always affected by the tip-electric field that induces a bending of the oxide bands during the spectral run. While this effect is negligible in ultrathin films, it might be substantial in thicker ones owing to the inefficient screening of the electric field at the oxide surface. For a six-trilayer film, for example, roughly $15 \%$ of the applied bias drops inside the ceria $\left(d=18 \AA, \varepsilon_{\mathrm{CeO}_{2}}=20\right)^{31}$ and induces a $350 \mathrm{mV}$ band shift with respect to the flat band situation. This bias dependence of the band position will contribute to the observed broadening in thicker films. We note that the intrinsic width of the ceria $\mathrm{f}$ band is further enhanced by the presence of vibrational sidebands that are excited via inelastic electron tunneling through the film. ${ }^{32}$

In any case, no filled states are detected in conductance spectra of defect-free oxide terraces and the $\mathrm{d} I / \mathrm{d} V$ signal increases only at the valence band onset. This observation is in agreement with the expected absence of occupied $4 \mathrm{f}$ states in stoichiometric ceria, where all cations are in the $4+$ oxidation state. However, the situation changes if the oxide film gets reduced and the $\mathrm{Ce} 4 \mathrm{f}$ states take up electrons from the desorbing $\mathrm{O}$ atoms. The resulting $\mathrm{d} I / \mathrm{d} V$ spectra are presented in the next section.

\subsection{Spectroscopy of point defects in the ceria surface}

Well-prepared oxide films exhibit only a low concentration of oxygen vacancies. Additional defects have thus been produced by bombarding the film with $50 \mathrm{eV}$ electrons (dosage $0.5 \mathrm{mC}) .{ }^{17}$ Under usual tip conditions, the resulting defects show up as single protrusions with $0.5 \AA$ apparent height and a sombrero shape (Fig. 3b). Only in atomically resolved images taken at positive bias (empty states), those maxima can be

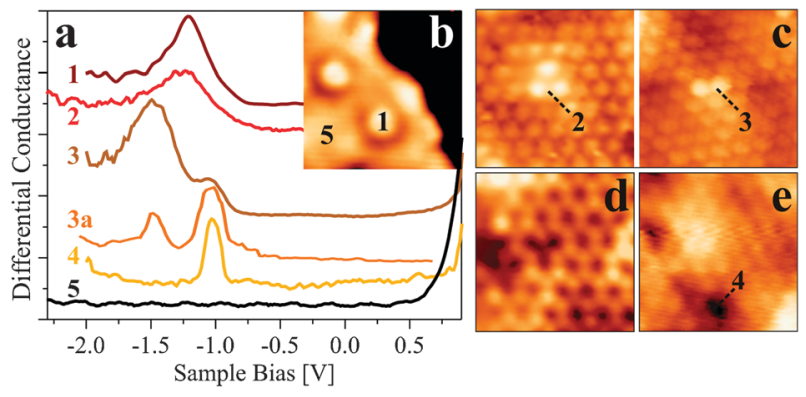

Fig. 3 (a) Filled-state spectra of different point defects in the ceria film (spectroscopy set point $+1.0 \mathrm{~V}$ ). The inset (b) shows a typical STM image of surface $\mathrm{O}$ vacancies, as they appear just below the onset of atomic resolution $\left(1.05 \mathrm{~V}, 6 \times 6 \mathrm{~nm}^{2}\right)$. Atomically resolved images of ceria point defects are shown on the right. (c) Fingerprint of two $\mathrm{O}$ vacancies in the Ce lattice that differ in the number of $\mathrm{Ce}^{3+}$ and $\mathrm{Ce}^{4+}$ ions around the defect. (d) Oxygen vacancy as it appears in the $\mathrm{O}$ sub-lattice imaged at negative bias. (e) Cerium vacancy imaged at positive bias $(2.0 \mathrm{~V})$. All images are $3 \times 3 \mathrm{~nm}^{2}$ in size.

further resolved into characteristic double or triple spots (Fig. 3c). At negative bias (filled states), the defects transform into atom-sized depressions with threefold symmetry (Fig. 3d). All those features are related to one and the same defect type, being assigned to surface oxygen vacancies in agreement with earlier experimental and theoretical work. ${ }^{14,15}$ The protrusions visible at positive bias mark the $\mathrm{Ce}^{4+}$ ions around the $\mathrm{O}$ defect that appear brighter because their $4 \mathrm{f}$ states are not confined by the $\mathrm{O} 2 \mathrm{p}$ orbitals anymore and expand towards the vacuum. ${ }^{24}$ The $4 \mathrm{f}$ orbitals of $\mathrm{Ce}^{3+}$ species, on the other hand, do not contribute to the tunneling, as they have shifted to higher energies due to the better screening of the $\mathrm{Ce}^{3+}$ core potential by the extra electron. The number of bright protrusions in atomically resolved images therefore reflects the distribution of $\mathrm{Ce}^{4+}$ versus $\mathrm{Ce}^{3+}$ ions in next neighbor positions to the vacancy (Fig. 3c). Whereas for triple protrusions, all nextneighbor cations are $\mathrm{Ce}^{4+}$ species and the $\mathrm{Ce}^{3+}$ ion pair occupies more distant sites, the defect is surrounded by two $\mathrm{Ce}^{4+}$ and one $\mathrm{Ce}^{3+}$ ion in the case of a double protrusion. The appearance of $\mathrm{O}$ defects in empty-state STM images is thus governed by regular $\mathrm{Ce}^{4+}$ ions next to the vacancy, while the position of the $\mathrm{Ce}^{3+}$ species is not revealed.

A second kind of atom-sized defects appears as $\sim 1 \AA$ deep depression in empty-state STM images (Fig. 3e). It is tentatively assigned to a vacancy cluster with one $\mathrm{Ce}$ and a few $\mathrm{O}$ ions being removed from the first oxide trilayer. Especially in highly annealed films, those holes are frequently observed and even become wider, indicating desorption of $\mathrm{Ce}-\mathrm{O}$ units at elevated temperature.

The creation of $\mathrm{O}$ vacancies in the ceria surface is inherently connected with the induction of filled states in the band gap, in accordance with established models for electron localization in the Ce $4 \mathrm{f}$ states. ${ }^{26,27}$ The energy of those levels is well-known from EELS and PES to be around $-1.5 \mathrm{eV}$, and spectral intensity in this region is often monitored to determine the ceria reduction state with non-local experimental techniques. ${ }^{22}$ A local detection of the filled $4 \mathrm{f}$ states with STM conductance spectroscopy is challenging due to the high spatial localization of the orbitals. To obtain sufficient spectral sensitivity, we have 
chosen a spectroscopy set-point directly at the onset of the empty $4 \mathrm{f}$ band at $1.0 \mathrm{~V}$, where the tip-sample distance is expected to be extremely short. ${ }^{33}$ The tip is then positioned above an $\mathrm{O}$ vacancy site, keeping in mind that the associated $\mathrm{Ce}^{3+}$ ions might not sit in a next neighbor position. ${ }^{27}$ Despite this uncertainty, we are able to detect $\mathrm{d} I / \mathrm{d} V$ maxima in the energy range where the filled $4 \mathrm{f}$ states occur in PES (Fig. 3a). ${ }^{22}$ The peaks become visible only in a small surface area and quickly vanish when displacing the tip by a few $\AA$, as expected for a highly confined orbital. Furthermore, the shape and bias position of the spectral features vary from defect to defect, as shown in Fig. 3a. While only a single peak at $-1.2 \mathrm{~V}$ is detected for the vacancy imaged as triple protrusion (No. 2), two peaks at $-1.1 \mathrm{~V}$ and $-1.5 \mathrm{~V}$ emerge for the double protrusion (No. 3). Interestingly, spectrum 3a that has been taken on a double protrusion as well shows the same maxima but with an opposite intensity ratio. We note that single and double-peak spectra are obtained for the sombrero-type defects whose atomic nature is not resolved (No. 1).

The origin of different spectral shapes for different $\mathrm{O}$ vacancies has been elucidated in recent DFT + U studies. ${ }^{24,27}$ The position of the filled $4 \mathrm{f}$ orbital was found to depend on the distance between the hosting $\mathrm{Ce}^{3+}$ ion and the associated vacancy site. The lowest $f$ state energy is adopted by the $\mathrm{Ce}^{3+}$ that is farthest from the defect, as lattice strain induced by the spacious $\mathrm{Ce}^{3+}$ is better accommodated in this case. In contrast, the $4 \mathrm{f}$ state moves towards $E_{\mathrm{F}}$ as the $\mathrm{Ce}^{3+}$ ion approaches the vacancy. The $4 \mathrm{f}$ related spectral feature is therefore split whenever the two $\mathrm{Ce}^{3+}$ ions are not in the same coordination shell of the associated defect and experience a different chemical environment. The peak splitting is hereby of the order of $0.1 \mathrm{eV}$ for two $\mathrm{Ce}^{3+}$ ions in adjacent coordination shells, let's say in a next and second next neighbor position of the defect, but reaches $0.5 \mathrm{eV}$ for a larger difference in the chemical environment. On this basis, the variability in the $\mathrm{d} I / \mathrm{d} V$ spectra shown in Fig. 3a can be explained. The two $\mathrm{Ce}^{3+}$ ions in No. 1 and 2 have comparable distances to the $\mathrm{O}$ defect and their $\mathrm{f}$ states show up as a single peak in the $\mathrm{d} I / \mathrm{d} V$ curve. The $\mathrm{Ce}^{3+}$ pair in No. 3 and $3 \mathrm{a}$, on the other hand, occupies rather different coordination shells and displays a clear splitting of the $4 \mathrm{f}$ related maximum. The different peak intensities in the doublet indicate the closeness of the tip to one or the other $\mathrm{Ce}^{3+}$ species during spectroscopy. In principle, a slight tip displacement should invert this intensity ratio, however the optimal tip position is hardly found in reality due to the unknown location of the two $\mathrm{Ce}^{3+}$ ions.

Also atom-sized holes in the Ce lattice exhibit filled electronic states inside the band gap (Fig. 3a, No. 4). The main difference to the spectra discussed before is the small peak width of only $200 \mathrm{mV}$, whereas the energy position remains comparable. We assign this spectral feature to filled $4 \mathrm{f}$ states in the gap as well, being created by an O-poor ceria composition around the defect site. Reduced Ce species develop for example if one $\mathrm{Ce}$ but more than two $\mathrm{O}$ ions are removed simultaneously from the surface trilayer. Such a scenario would be compatible with the dark appearance of the defect in empty-state images that suggests a Ce vacancy. It would also explain the small line width, as the extra electrons might localize in deeper layers, where the effect of tip-induced line broadening, as discussed in
Section 3.1, is less pronounced. However, a comprehensive interpretation of such a spectral feature remains difficult as long as the atomic configuration of the defect cannot be confirmed.

\subsection{Spectroscopy of line defects in the ceria surface}

In the last section of this paper, we want to discuss the spectral fingerprints of ceria line defects. Step edges are the most abundant defect type, although dislocation lines and grain boundaries have been identified as well. ${ }^{17}$ Especially the latter types develop spontaneously in many thin films, as they provide an effective means to compensate the lattice mismatch between the oxide film and the metal support. ${ }^{8}$ Line defects are known to play a crucial role for the adsorption and chemical behavior of ceria and have been identified as active regions for charge transfer and nucleation processes. ${ }^{17,21,34}$ This enhanced reactivity is related to a deviating structure of the line defects, but also reflects their high susceptibility for reduction processes. We have analyzed the electronic properties of step edges and grain boundaries for non-reduced ceria films, using again STM conductance spectroscopy.

A selection of defect lines, e.g. boundaries to multilayerdeep holes and simple step edges, is displayed in Fig. 4 together with their $\mathrm{d} I / \mathrm{d} V$ spectra. Common to all $1 \mathrm{D}$ defects is the occurrence of gap states below $E_{\mathrm{F}}$, which occur again in the energy range of the filled $4 \mathrm{f}$ states. For example, peaks between -1.3 and $-1.6 \mathrm{~V}$ emerge along the brim of an oxide hole, with their positions depending on the actual morphology at the spectroscopy point (Fig. 4a, No. 1 and 2). The $\mathrm{d} I / \mathrm{d} V$ maximum disappears when the tip moves away from the brim, as seen in spectrum 4 that has been taken inside the hole. On trilayer-high steps, the conductance maxima mainly occur at distinct low-coordinated sites, e.g. kink and corner positions, but vanish in other sections (Fig. 4d). As already stated, the peak position suggests again a relation to the partly filled $4 \mathrm{f}$ states localized at the $\mathrm{Ce}^{3+}$ ions. Apparently, filled gap states are not only present in artificially reduced oxide films, but spontaneously develop in the vicinity of oxide line defects.

The spatial localization of filled $\mathrm{f}$ orbitals at a line defect has been deduced from spectral series taken with $2.5 \AA$ intervals

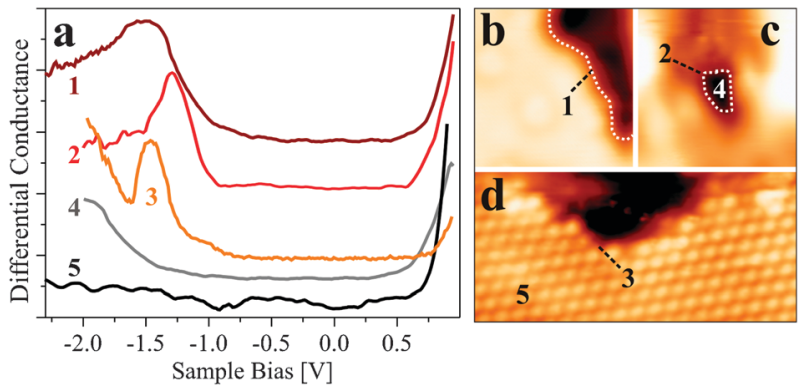

Fig. 4 (a) Filled-state spectra of different line defects in the ceria film (set point $+1.0 \mathrm{~V}$ ). STM images displaying the corresponding tip positions for spectroscopy are shown in the right. (b and c) Positions along the ceria brim next to small holes in the oxide film $(1.05 \mathrm{~V}$, $6 \times 6 \mathrm{~nm}^{2}$ ). (d) Trilayer-high ceria step edge imaged with atomic resolution $\left(1.6 \mathrm{~V}, 7.5 \times 3.5 \mathrm{~nm}^{2}\right)$. 


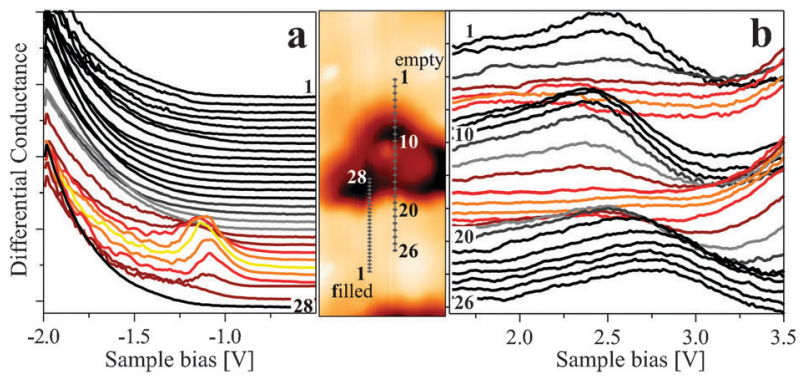

Fig. 5 Series of $\mathrm{d} I / \mathrm{d} V$ spectra taken across the trilayer-deep hole in the $\mathrm{CeO}_{2}$ film that is shown in the central panel $\left(2.2 \mathrm{~V}, 22 \times 9 \mathrm{~nm}^{2}\right)$. Curves on the left and right sides display the filled and empty states, respectively. At the ceria step edge, filled $f$ states appear while the empty $4 \mathrm{f}$ band loses intensity and the conduction band moves into the spectral window. See text for details.

across a step edge (Fig. 5a). As expected, the characteristic $-1.2 \mathrm{~V}$ peak becomes visible just in a couple of spectra taken directly at the step position, while only the band gap is revealed in the other curves. Already a $5 \AA$-displacement of the tip from the edge is sufficient to suppress the peak, giving a rough measure of the spatial extension of filled $4 \mathrm{f}$ orbitals. The corresponding empty-state series, taken with $5 \AA$ step size across two nearby edges, reveals a more complex behavior (Fig. 5b). The dominant spectral feature is now the empty $4 \mathrm{f}$ band at around $2.5 \mathrm{~V}$, being visible in the flat surface regions between the steps. Directly at the edge positions (bright spectra), the $4 \mathrm{f}$ band flattens while new $\mathrm{d} I / \mathrm{d} V$ intensity moves into the spectral range at higher bias. This evolution can be explained by a downshift of the conduction band above certain step edges that gives rise to enhanced $\mathrm{d} I / \mathrm{d} V$ intensity at $3.5 \mathrm{~V}$. The availability of the ceria conduction band for tunneling now prompts the spectroscopy set-point to move away from the surface, which decreases the spectral sensitivity and renders the localized $4 \mathrm{f}$ band invisible. The true information contained in this $\mathrm{d} I / \mathrm{d} V$ series is therefore the downshift of the conduction band at the step edge, while the suppression of the $4 \mathrm{f}$ states is only an artifact of the measurement. ${ }^{35} \mathrm{We}$ tentatively assign this band shift to an uncompensated electrostatic dipole that occurs at certain ceria steps. A more detailed discussion of this topic will be given in a forthcoming publication. $^{36}$

The presence of filled gap states along ceria line defects is a direct consequence of the inherent non-stoichiometry of the respective oxide regions. Every line defect requires a cut through the regular $\mathrm{O}-\mathrm{Ce}-\mathrm{O}$ trilayer structure, where not all atomic planes can be cut at once and incomplete layers with a perturbed O-to-Ce ratio may develop. The resulting oxide composition will mostly be oxygen-poor, as the $\mathrm{Ce}^{4+}$ ions cannot stabilize more $\mathrm{O}$ species than in $\mathrm{CeO}_{2}$. This oxygen deficiency gives rise to the presence of excess electrons that become trapped again in the $4 \mathrm{f}$ states of adjacent $\mathrm{Ce}$ ions. The fact that the reduced ions remain close to the step edges and do not dissolve into the oxide bulk is explained by a facile lattice relaxation when the spacious $\mathrm{Ce}^{3+}$ occupy low-coordinated sites. Although the accumulation of $\mathrm{Ce}^{3+}$ ions along ceria line defects was predicted by theory, ${ }^{19}$ it has now been confirmed in a direct and spatially resolved experiment.

\section{Summary}

We have demonstrated that STM conductance spectroscopy is a suitable method to probe the local electronic structure of $\mathrm{CeO}_{2}(111)$ films grown on a $\mathrm{Ru}(0001)$ support. In contrast to earlier experiments on transition and rare-earth metal oxides, ${ }^{8}$ the $\mathrm{d} I / \mathrm{d} V$ spectra reveal not only the size and position of the fundamental band gap, but also display 4f-type gap states localized at the $\mathrm{Ce}$ ions. The spatially confined $4 \mathrm{f}$ orbitals are accessible to the experiment, because no other states are available in the oxide band gap to sustain the preset tunnel current. Still, only the empty $4 \mathrm{f}$ band features enough transport capacity to stabilize the STM feedback loop (at 5 pA), while the filled $4 \mathrm{f}$ states can only be probed in the spectroscopy mode with the feed-back turned off.

The feasibility of $f$ state spectroscopy in ceria opens various new options to explore this fascinating material system; only a few of them could be realized here. The technique provides insight into the energy position and width of the empty $\mathrm{Ce} 4 \mathrm{f}$ band that is accessible only to inverse PES otherwise. ${ }^{23}$ Filledstate spectroscopy with the STM enables the detection of occupied $4 \mathrm{f}$ levels being induced by $\mathrm{O}$ vacancies. Although this spectral fingerprint has long been used to judge the overall reduction state of ceria, it can now be probed locally and correlated with the atomic-scale surface morphology detected in parallel. With this approach, the electronic perturbations induced by different defect types can be analyzed with unmatched spatial resolution. We have shown that point and line defects generate similar electronic signatures in the oxide band gap and that $\mathrm{Ce}^{3+}$ ions are not only present in artificially reduced but also in pristine films. This finding emphasizes the special role of step edges and grain boundaries for adsorption and nucleation processes on stoichiometric oxide surfaces. In future, we plan to extend our studies towards the exploration of adsorbate-induced changes in the ceria electronic structure, focusing on charge-transfer processes between suitable ad-species and the reducible oxide. From such experiments, we expect a better understanding of key processes taking place during a chemical reaction.

\section{Acknowledgements}

We are grateful for financial support from the DFG within the Cluster of Excellence 'UniCat'.

\section{References}

1 H.-J. Freund, H. Kuhlenbeck and V. Staemmler, Rep. Prog. Phys., 1996, 59, 283.

2 J. Kramer, C. Tegenkamp and H. Pfnür, Phys. Rev. B: Condens. Matter, 2003, 67, 235401.

3 M. Anpo, Y. Yamada, Y. Kubokawa, S. Coluccia, A. Zecchina and M. Che, J. Chem. Soc., Faraday Trans., 1988, 184, 751; S. Stankic, M. Muller, O. Diwald, M. Sterrer, E. Knözinger and J. Bernardi, Angew. Chem., Int. Ed., 2005, 44, 4917.

4 M. Sterrer, E. Fischbach, T. Risse and H.-J. Freund, Phys. Rev. Lett., 2005, 94, 186101.

5 G. Haas, A. Menck, H. Brune, J. V. Barth, J. A. Venables and K. Kern, Phys. Rev. B: Condens. Matter, 2000, 61, 11105.

6 M. Sterrer, M. Heyde, M. Nowicki, N. Nilius, T. Risse, H.-P. Rust, G. Pacchioni and H.-J. Freund, J. Phys. Chem. B, 2006, $110,46$. 
7 M. Schmid, M. Shishkin, G. Kresse, E. Napetschnig, P. Varga, M. Kulawik, N. Nilius, H.-P. Rust and H.-J. Freund, Phys. Rev. Lett., 2006, 97, 046101.

8 N. Nilius, Surf. Sci. Rep., 2009, 64, 595.

9 N. Nilius, M. Kulawik, H.-P. Rust and H.-J. Freund, Phys. Rev. B: Condens. Matter, 2004, 69, 121401.

10 A. Trovarelli, Catalysis by Ceria and Related Materials, World Scientific Publishing, 2002.

11 Q. Fu, H. Saltsburg and M. Flytzani-Stephanopoulos, Science, 2003, 301, 935.

12 F. Yang, J. Graciani, J. Evans, P. Liu, J. Hrbek, J. Sanz and J. A. Rodriguez, J. Am. Chem. Soc., 2011, 133, 3444; G. N. Vayssilov, Y. Lykhach, A. Migani, T. Staudt, G. P. Petrova, N. Tsud, T. Skála, A. Bruix, F. Illas, K. C. Prince, V. Matolin, K. M. Neyman and J. Libuda, Nat. Mater., 2011, 10, 310.

13 H. Norenberg and G. A. D. Briggs, Surf. Sci., 1999, 424, L352.

14 F. Esch, S. Fabris, L. Zhou, T. Montini, C. Africh, P. Fornasiero, G. Comelli and R. Rosei, Science, 2005, 309, 752.

15 S. Torbrügge, M. Reichling, A. Ishiyama, S. Morita and Ó. Custance, Phys. Rev. Lett., 2007, 99, 056101; S. Torbrügge, M. Cranney and M. Reichling, Appl. Phys. Lett., 2008, 93, 073112.

16 D. C. Grinter, R. Ithnin, C. L. Pang and G. Thornton, J. Phys. Chem. C, 2010, 114, 17036.

17 J.-L. Lu, H.-J. Gao, S. Shaikhutdinov and H.-J. Freund, Catal. Lett., 2007, 114, 8; J.-L. Lu, H.-J. Gao, S. Shaikhutdinov and H.-J. Freund, Surf. Sci., 2006, 600, 5004.

18 M. Nolan, S. C. Parker and G. W. Watson, Surf. Sci., 2005, 595, 223.

19 C. Loschen, S. T. Bromley, K. M. Neyman and F. Illas, J. Phys. Chem. C, 2007, 111, 10142; M. M. Branda, C. Loschen, K. M. Neyman and F. Illas, J. Phys. Chem. C, 2008, 112, 17643.

20 M. Fronzi, A. Soon, B. Delley, E. Traversa and C. Stampfl, J. Chem. Phys., 2009, 131, 104701.

21 J. A. Farmer and C. T. Campbell, Science, 2010, 329, 933.

22 A. Pfau and K. D. Schierbaum, Surf. Sci., 1994, 321, 71.

23 H. R. Moser, B. Delley, W. D. Schneider and Y. Baer, Phys. Rev. B: Condens. Matter, 1984, 29, 2947; E. Wuilloud, B. Delley, W. D. Schneider and Y. Baer, Phys. Rev. Lett., 1984, 53, 202.
24 J.-F. Jerratsch, X. Shao, N. Nilius, H.-J. Freund, C. Popa, M. V. Ganduglia-Pirovano, A. M. Burow and J. Sauer, Phys. Rev. Lett., 2011, in press.

25 D. R. Mullins, P. V. Radulovic and S. H. Overbury, Surf. Sci., 1999, 429, 186; D. R. Mullins, L. Kundakovic and S. H. Overbury, J. Catal., 2000, 195, 169.

26 S. Fabris, G. Vicario, G. Balducci, S. de Gironcoli and S. Baroni, J. Phys. Chem. B, 2005, 109, 22860.

27 M. V. Ganduglia-Pirovano, J. L. Da Silva and J. Sauer, Phys. Rev. Lett., 2009, 102, 026101.

28 L. Berbil-Bautista, T. Hänke, M. Getzlaff, R. Wiesendanger, I. Opahle, K. Koepernik and M. Richter, Phys. Rev. B: Condens. Matter, 2004, 70, 113401.

29 Y. Q. Xue, S. Datta, S. Hong, R. Reifenberger, J. I. Henderson and C. P. Kubiak, Phys. Rev. B: Condens. Matter, 1999, 59, R7852; M. Grobis, A. Wachowiak, R. Yamachika and M. F. Crommie, Appl. Phys. Lett., 2005, 86, 204102.

30 N. Nilius, T. M. Wallis and W. Ho, Phys. Rev. Lett., 2003, 90, 046808.

31 A. S. Nowick, A. V. Vaysleyb and I. Kuskovsky, Phys. Rev. B: Condens. Matter, 1998, 58, 8398.

32 J. Repp, G. Meyer, S. Paavilainen, F. E. Olson and M. Persson, Phys. Rev. Lett., 2005, 95, 225503.

33 Note that ceria films are insulators and scanning is impossible for bias voltages between the valence band onset $(-2.5 \mathrm{~V})$ and the onset of the empty $4 \mathrm{f}$ band $(1.0 \mathrm{~V})$.

34 N. J. Castellani, M. A. Branda, K. M. Neyman and F. Illas, J. Phys. Chem. C, 2009, 113, 4948; N. C. Hernandez, R. GrauCrespo, N. H. de Leeuw and J. F. Sanz, Phys. Chem. Chem. Phys., 2009, 11, 5246.

35 The suppression of the empty $4 \mathrm{f}$ band above the step edge is also compatible with the distinct spectral signature of the $\mathrm{Ce}^{3+}$ ions, whose $f$ states shift to higher energy due to the better screening of the core potential by the extra electron. However, we expect this effect to be covered by the large number of regular $\mathrm{Ce}^{4+}$ ions along the edge that still dominate the $\mathrm{d} I / \mathrm{d} V$ response.

36 N. Nilius, J.-F. Jerratsch, M. Baron, X. Shao, J.-L. Lu, S. Shaikhutdinov, H.-J. Freund, S. M. Kozlov, A. Migani, F. Viñes and K. M. Neyman, in preparation. 